Characterization of BG28 and KG3 filter glass for Drive Diagnostic Attenuators

R. H. Page, T. Weiland, J. Folta

December 5, 2007 
This document was prepared as an account of work sponsored by an agency of the United States government. Neither the United States government nor Lawrence Livermore National Security, LLC, nor any of their employees makes any warranty, expressed or implied, or assumes any legal liability or responsibility for the accuracy, completeness, or usefulness of any information, apparatus, product, or process disclosed, or represents that its use would not infringe privately owned rights. Reference herein to any specific commercial product, process, or service by trade name, trademark, manufacturer, or otherwise does not necessarily constitute or imply its endorsement, recommendation, or favoring by the United States government or Lawrence Livermore National Security, LLC. The views and opinions of authors expressed herein do not necessarily state or reflect those of the United States government or Lawrence Livermore National Security, LLC, and shall not be used for advertising or product endorsement purposes.

This work performed under the auspices of the U.S. Department of Energy by Lawrence Livermore National Laboratory under Contract DE-AC52-07NA27344. 
NIF0114511

\title{
Characterization of BG28 and KG3 filter glass for Drive Diagnostic Attenuators
}

\author{
Ralph H. Page \\ V-division, PAT, LLNL \\ Tim Weiland \\ Optical Sciences Laser facility, NIF, LLNL \\ James Folta \\ Final Optics Systems, NIF, LLNL
}

4 Oct 2007

\begin{abstract}
BG28 and KG3 filter glasses were tested for use as attenuators in the NIF drive diagnostic (DrD) systems. Tests were performed in the Optical Sciences Laser facility with a $351 \mathrm{~nm}, 2$-step, 3-nsec pulse at fluences ranging up to $\sim 1 \mathrm{~J} / \mathrm{cm}^{2}$. Single-shot measurements showed no solarization when the samples were allowed to relax for a week after exposure. KG3 filters exhibited no luminescence and no transient pulse distortion. BG28 filters luminesced appreciably and imposed a "droop" (similar to "square-pulse distortion") on the signals. The droop parameter is estimated at $0.50 \pm 0.11 \mathrm{~cm}^{2} / \mathrm{J}$. Droop is explained in terms of known copper-doped-glass spectroscopy and kinetics (buildup of triplet-state populations, with excited-state absorption). Simulation of the distortion $(\sim 1.6 \%)$ expected on a $1.8 \mathrm{MJ}$ Haan pulse led to a minor redesign of the Drive Diagnostic with reduced fluence on the BG28 filters to reduce the droop distortion to $0.5 \%$.
\end{abstract}

Authors' Note: Several months after this study, the air side drive diagnostic was modified because the beam splitting turning mirrors (WM8 and WM14) had reflectance specifications which could not be achieved in practice. The turning mirror reflectances were increased, which required the first BG28 filter to be changed to NG4 to avoid nonlinear absorption effects. The NG4 filter will have to be changed for the system to be used at $526 \mathrm{~nm}$. The new design change is in lieu of that described in section $\mathrm{V}$.

\section{Introduction}

To achieve ignition in an ICF experiment, the power balance, pulse shape, and timing of each of the 192 drive laser beams must be carefully controlled. Hence beam power must be measured with a well-calibrated, high-bandwidth diagnostic. On NIF, this capability is met with the "Drive Diagnostics," one function of which is to measure the laser pulse shapes. This is done by sampling the beams with the Grating Debris Shields (GDSs), and relaying and attenuating the beam samples to photodiodes monitored with fast oscilloscopes.

Keywords for search: drive diagnostic power sensor, BG28, KG3, NG4, attenuators, filters, filter glass, nonlinear absorption 
Many specifications pertain to the Drive Diagnostic - the ones most important for the sake of this memorandum are the amount of attenuation and the degree of distortion induced in pulse shape. To illustrate the orders of magnitude involved, consider that a reasonable peak illumination for a photodiode is $\sim 1$ Watt, whereas a beamline delivering $\mathrm{a} \sim 10 \mathrm{~kJ}$ pulse in $\sim 10 \mathrm{nsec}$ possesses a peak power of $\sim 1 \mathrm{TW}$. Thus a factor of $\sim 10^{12}$ attenuation is needed; the GDS provides a factor $\sim 10^{3}$, and the remaining 9 orders of magnitude are derived from partial reflectors and attenuating filters. During a laser pulse, we generally seek a photodiode distortion level below $\sim 1 \%$.

A picture of the components of the air-side Drive Diagnostic is shown in Figure 1. Light picked off by the GDS passes through the vacuum-side assembly and through a vacuum window, then reflects from a pair of partial reflectors - one each working nominally at $\mathrm{S}$ and $\mathrm{P}$ polarization. Then it passes through a BG28 filter (the main topic of this memo), a concentrating lens, and a pair of filters on filter wheels en route to the fiber launch homogenizer and into an optical fiber whose far end is interfaced to a high-bandwidth photodiode. The reflectances and attenuation values were chosen to offer the requisite diminution of the signal strength while affording sufficient dynamic range to cover different conditions of pulse energy, pulse shape, etc.

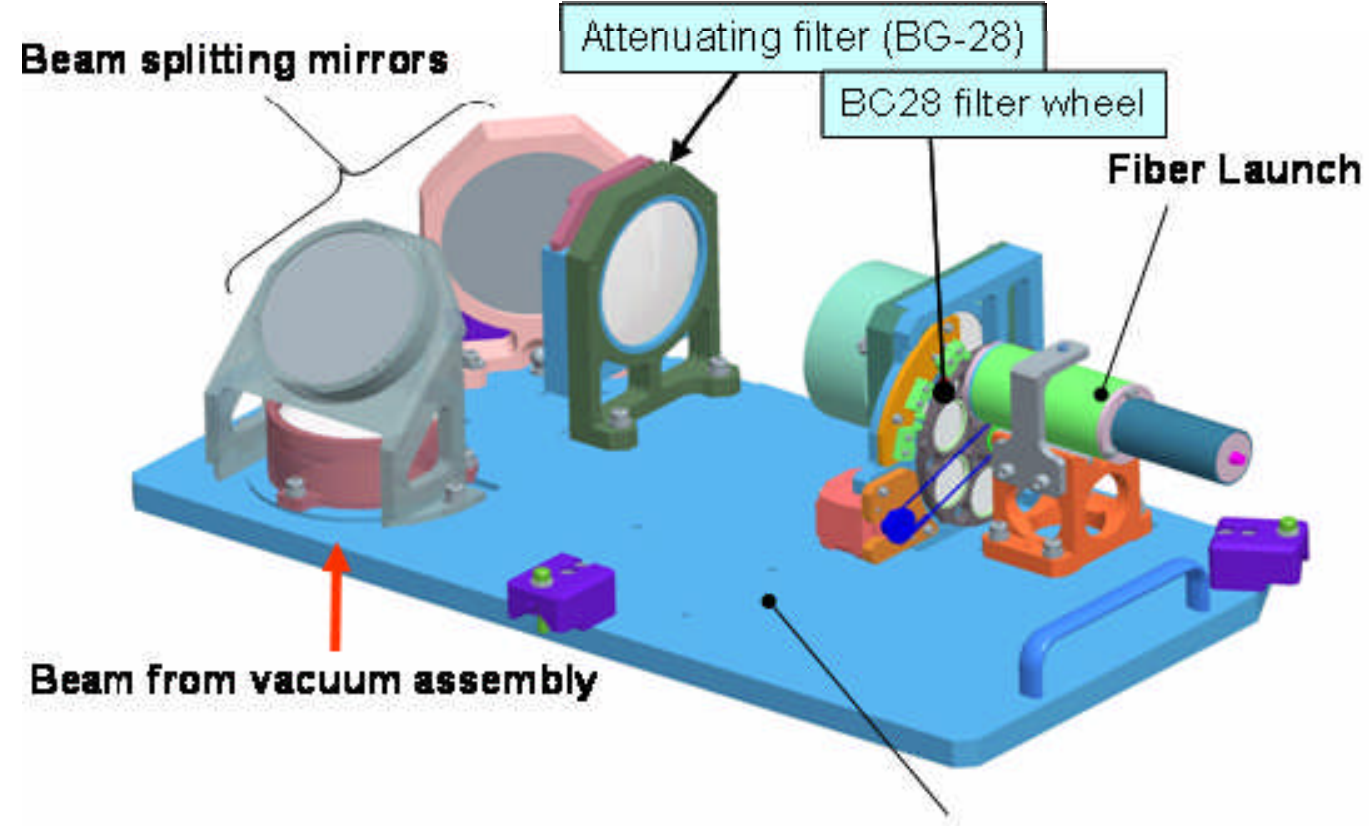

Fig. 1. Layout of the air-side Drive Diagnostic. Light from the vacuum side reflects from a pair of partial reflectors, is attenuated with BG28 filters, and homogenized in the fiber launch.

The filter glasses considered for use in the Drive Diagnostic include Schott BG28, KG3, and NG-4. Typical (catalog) spectra for these filters are shown in Figure 2; note that the thicknesses and attenuation values actually deployed will be different. Vertical lines on the spectra indicate wavelengths of the $1053 \mathrm{~nm}$ fundamental and 527 and $351 \mathrm{~nm}$ harmonics propagating on the beamline. (The wavelength of chief interest, selected by the GDS, will be $351 \mathrm{~nm}$.) Use of the BG28 filter, whose attenuation is substantially 
equivalent at the two shorter wavelengths, allows the Drive Diagnostic to be used at 527 $\mathrm{nm}$ if needed. The transmission of the first filter is designed to be 0.03 (i.e. lower than shown in the figure). Downstream filters would also be various thicknesses of BG28. KG3 is "heat-absorbing glass" that might be desired if $1053 \mathrm{~nm}$ leakage into the diagnostic is a problem. NG-4 glasses are simply "neutral-density filters" destined for the calorimeter; their job is to convert light into heat.
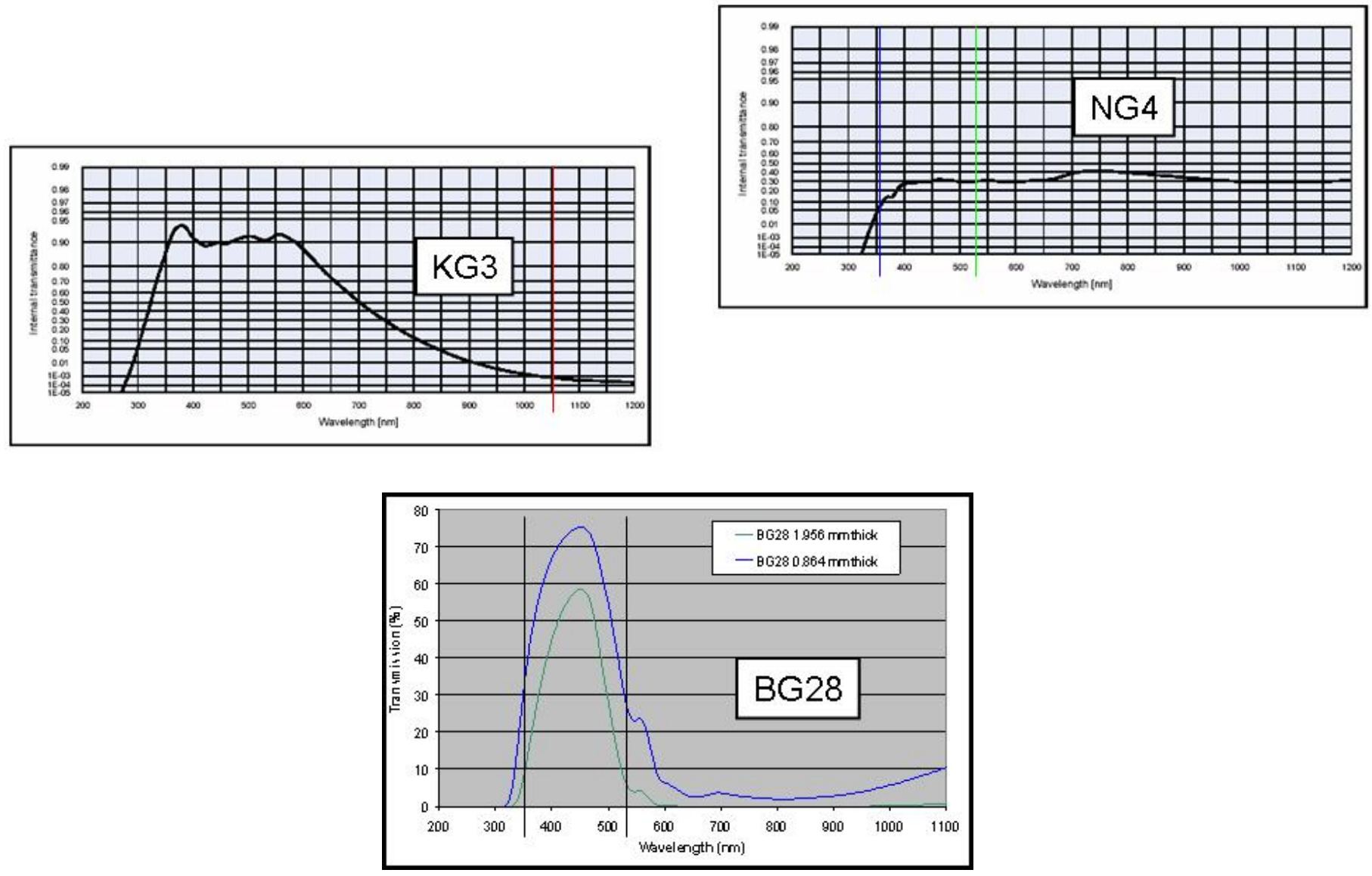

Fig. 2. Transmission spectra [stock thickness] for Schott glasses BG28, KG3, and NG-4, to be used in the Drive Diagnostic. BG28 was selected for its similar transmittance values at 527 and $351 \mathrm{~nm}$. It will provide most of the attenuation. KG3 is for IR blocking, and NG-4 is used in the calorimeter on the vacuum side.

Use of filter glasses raises concerns about time-dependent changes in their transmission during the pulse duration. Slow changes (drift) can be caused by "solarization," [1] which is not a serious problem if the filters are located downstream of the calorimetry. Transmission changes during a few-nsec laser pulse are of great concern-bleaching and/or nonlinear absorption will degrade pulse fidelity, reducing ability to measure beam power. Of course, existence of solarization is evidence that chemical changes are occurring, making us wonder if transient effects are also present. In fact, solarization and nonlinear-optical effects in glasses have been an important topic for many years. [2 - 5] 
Prior to this study, the maximum design fluence at the first BG28 filter was $0.04 \mathrm{~J} / \mathrm{cm}^{2}$. Compared with fluences frequently found on mirrors and in laser gain media, this may seem small. However, BG28 filters tested at higher fluence had exhibited unwanted behavior, causing concern. A filter used at $1053 \mathrm{~nm}$ in a diagnostic path on NIF exhibited a phenomenon called "measles," in which the pattern of deformable-mirror actuators was evident in a diagnostic image. [6] This was attributed to some phase-toamplitude conversion coupled with non-linear absorption in the BG28. In another case, tests (Figure 3) by John Adams et al. [7] at $355 \mathrm{~nm}$ showed that solarization in BG28 did indeed occur-tens of $\mathrm{J} / \mathrm{cm}^{2}$ changed the transmittance noticeably. Solarization in copper-containing filters has been reported [8,9] and the Schott catalog specifically mentions its occurrence in BG28. [10]

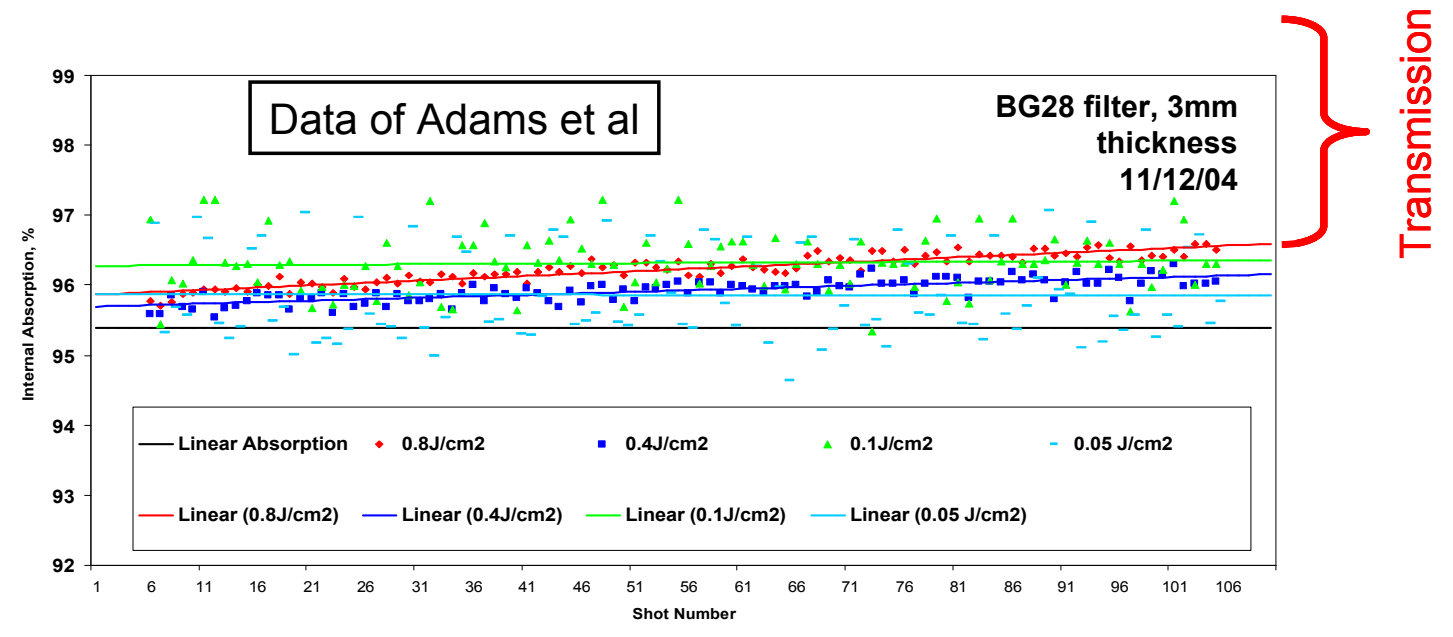

Fig. 3. Data of Adams et al. [7] on solarization of BG28 at $355 \mathrm{~nm}$. Gaussian pulses 7.2 nsec wide were used in the experiments. Pulse transmissions were measured with calorimeters; at the lower fluences, the signal-to-noise ratio was therefore not as good as at 0.4 and $0.8 \mathrm{~J} / \mathrm{cm}^{2}$, where the effect is obvious.

\section{Experimental setup}

Transient pulse distortion and solarization of the filters was measured with the Optical Sciences Laboratory (OSL) laser. The notional plan for our experiments is shown in Figure 4. The measurements were performed from $19-30$ April 07. A series of filters was irradiated at various fluences with well-defined pulse shapes. In principle, to test for solarization, absorption spectra would have been recorded shortly after the illumination, and then at later times (one day, and one week) to see if the absorption spectrum relaxed to its pre-shot condition. (We obtained spectra of virgin filters, and one week after irradiation. See below.) Transient effects were monitored with photodiodes recording the incident ("reference") and transmitted ("signal") waveforms. A further check on nonlinear-transmission effects came from calorimeters, as in the Adams, et al. experiment. 


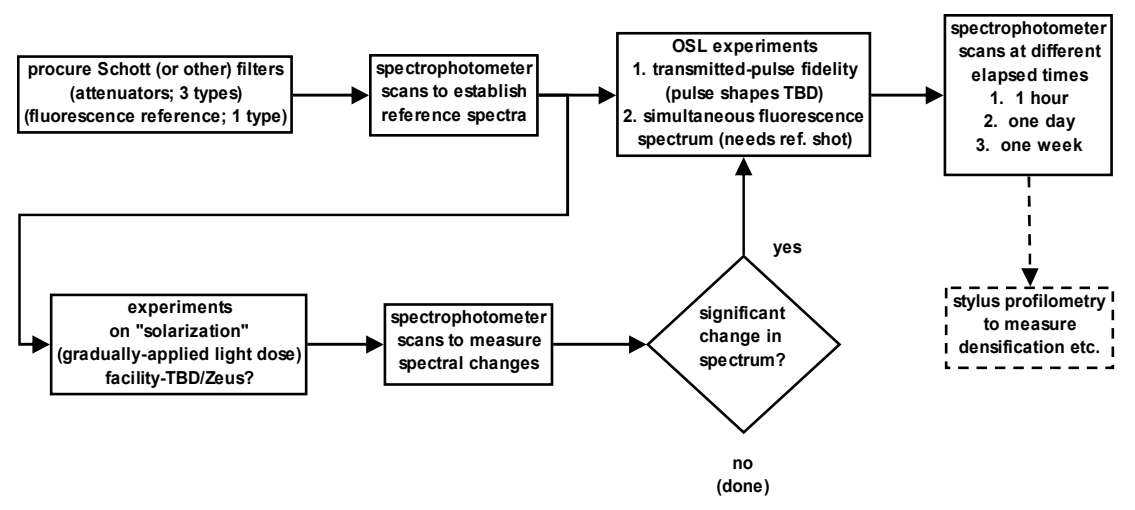

Establishing stable filter operating regime with good transmitted-pulse fidelity is main goal

Fig. 4. Outline for filter test plan, incorporating tests for nonlinear transmission and for solarization. Fluorescence monitoring adds to the ability to diagnose kinetic phenomena.

Figure 5 is a diagram of the layout we created on the "Multi-Wavelength Table" in OSL. Some lenses were used for imaging and for controlling the spot size on the filters. Also, the photodiodes (Hamamatsu 1351 vacuum photodiodes) had UV-transmitting UG-11 filters and attenuators to control the signal level and block stray light. Although not a mandatory feature of the experiments, we included a spectrometer and photodiode to detect fluorescence from the edges of the filters; these were shielded from the $351 \mathrm{~nm}$ pump light with GG-10 filters. Normally filters that do not fluoresce are preferred; luminescence is evidence of excited-state populations that can lead to bleaching or excited-state absorption. To calibrate the fluorescence-detection channel, we used an OG-550 filter, which produces orange-red fluorescence. Its quantum yield is estimated at a few percent, based on the work of Penzkofer [11] and others. A camera acquired spot images at a plane conjugate to that of the filter under test. Its magnification was calibrated prior to data acquisition.

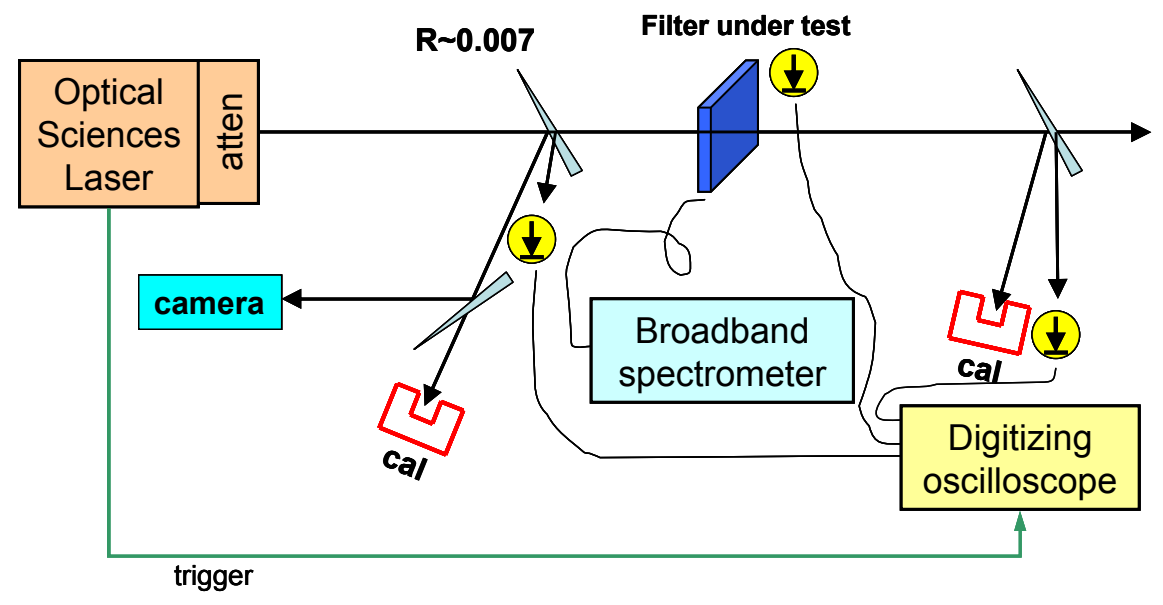

Fig. 5. Simplified diagram of experiment set up on the Multi-Wavelength Table in OSL. Fast photodiodes monitored filter transient response; pyroelectric calorimeters measured solarization and aided the photodiode calibration; luminescence detection checked for excited-state population. 
Filter samples we procured for this experiment included:

- NG4 filter glass (.042” thick,) CVI ANG-57-2X2

- OG550 filter glass (.120” thick,) Newport FSQ-OG550

- KG3 filter glass (.120” thick, ) Newport FSQ-KG3

- BG28 filter glass (.125" thick,) Melles Griot 03FCG025

The suite of filters and fluences selected for testing is shown in Figure 6.

\begin{tabular}{|l|l|l|l|l|}
\hline Glass & Function & Shot \# & $\begin{array}{l}\text { Fluence } \\
\left(\mathrm{J} / \mathrm{cm}^{2}\right)\end{array}$ & Remarks \\
\hline BG28 & Diag. power sensor & 1 & .004 & Did not do (too low) \\
\hline & & 2 & .04 & Maximum design fluence \\
\hline & & 3 & .4 & \\
\hline & & 4 & 1 & \\
\hline KG3 & Alternate / IR block & 1 & .004 & Did not do (too low) \\
\hline & & 2 & .04 & Maximum design fluence \\
\hline & & 3 & .4 & \\
\hline & & 4 & 1 & \\
\hline OG550 & Fluorescence ref. & 1 & Low TBD & Need to get a signal \\
\hline & & $(2)$ & TBD & \\
\hline
\end{tabular}

Fig 6. Nominal schedule of filters to be tested and fluences to be applied. Maximum fluences extended well above the nominal operating point of $0.04 \mathrm{~J} / \mathrm{cm}^{2}$. In general, a few shots were done at each fluence listed.

As key diagnostic components, the BG28 filters received the most extensive testing, at fluences ranging up to 25 times higher than their nominal $0.04 \mathrm{~J} / \mathrm{cm}^{2}$ operating point. (As it happened, the dynamic range of OSL was not so high that we could conveniently apply pulses below $0.04 \mathrm{~J} / \mathrm{cm}^{2}$.) KG3 filters are also potential candidates for use in the diagnostic path and were tested at several fluences. The OG-550 filters provided a fluorescence standard and received a few shots. Since the NG-4 filters were destined only for calorimeter use, testing for nonlinear-optical response was not deemed necessary. In addition, past studies have shown that NG-4 filters do not exhibit nonlinear absorption at the fluences of interest.

Discrimination among various types of filter nonlinearities can be facilitated with a test pulse having more than one "plateau" in its intensity-vs-time profile (see Figure 7.) Twophoton absorption, for example, could act quickly compared with the pulse width, and therefore preferentially attenuate the stronger parts of the pulse. Other kinetic effects (bleaching, square-pulse distortion) might act gradually on the pulse's time-scale. The "two-step" pulse used in this study (Fig. 7(b)) represents a compromise between the extremes of pulse shape and duration potentially used on NIF. Its 3 nsec duration is intermediate. In general, it is important for the first "knee" to be well-defined so as to furnish a clean reference point for comparing incident and transmitted pulses. Because of nonlinearities in frequency tripling used to generate the $351 \mathrm{~nm}$ test beam, the height of 
the knee with respect to the overall maximum declined as the energy was reduced. Also, the low dynamic range and modest signal-to-noise ratio made testing with ignitionrelevant (i.e. "Haan") pulse shapes unrealistic.
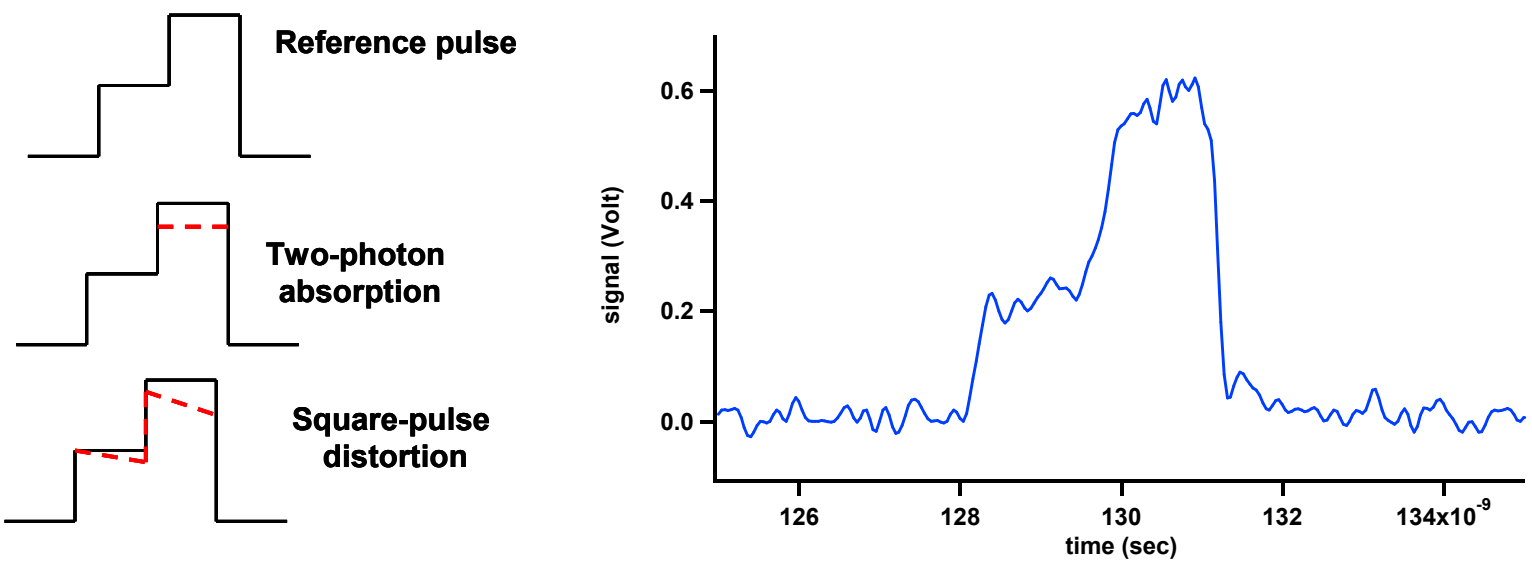

Fig. 7. (a) Use of a "Two-step" pulse can discriminate between effects of two-photon absorption and square-pulse distortion. (b) Sample pulse produced in OSL, of duration 3 nsec.

\section{Results}

A digitizing camera image of one of the beam profiles used (shot 7043014) is shown in Figure 8(a). Statistical processing yielded the differential and cumulative fluence distributions in Figure 8(b.) Although "hot spots" are evident, for the sake of simplicity we used a "top hat" approximation to describe this beam. The effective spot area was estimated as $\mathrm{A}_{\mathrm{TH}}=\mathrm{E}_{\text {pulse }} / \mathrm{F}_{\mathrm{avg}} \sim 0.35 \mathrm{~cm}^{2} ; \mathrm{A}_{\mathrm{TH}}$ is the area of the top-hat, $\mathrm{E}_{\text {pulse }}$ is the pulse energy recorded by the calorimeter, and $\mathrm{F}_{\text {avg }}$ is the average fluence determined with the image-processing routine.
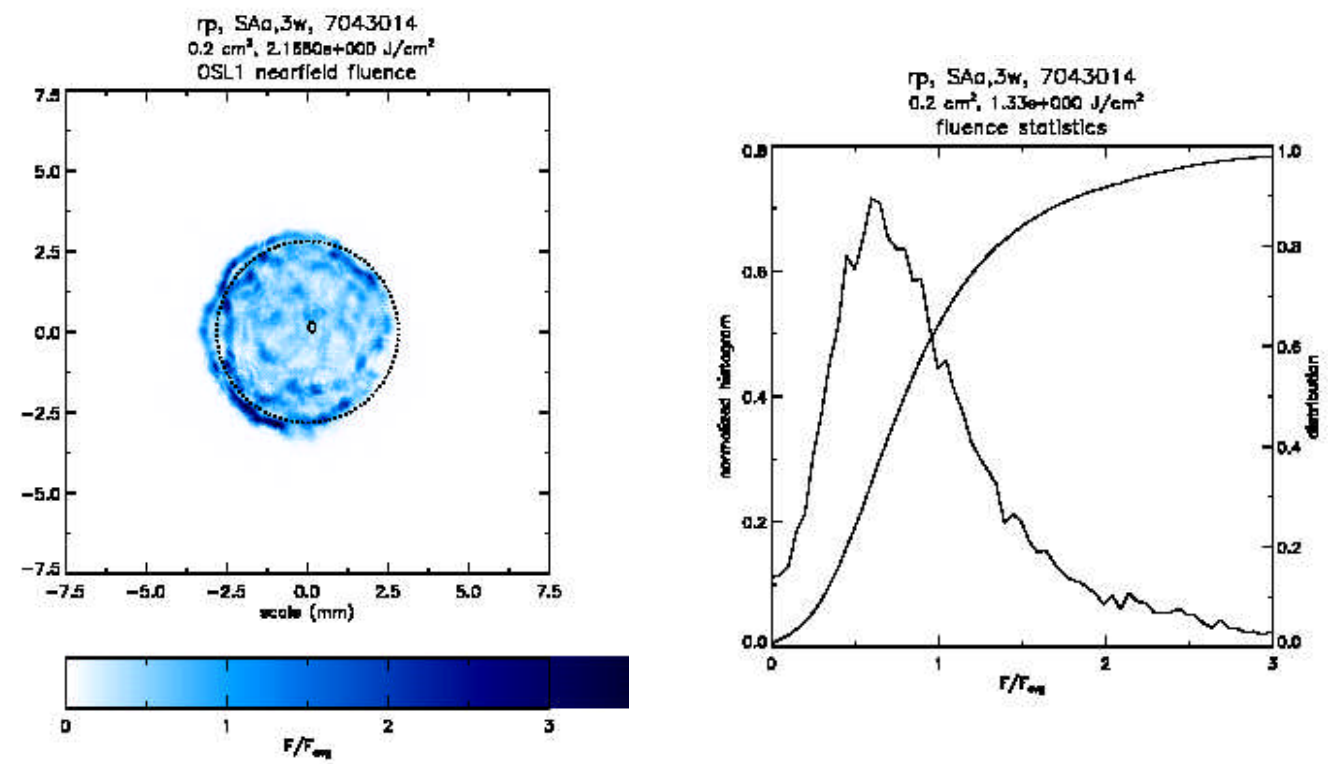

Fig. 8. Test beam fluence map (a) and statistics (b) determined from post-processing. The image was obtained at a plane conjugate to that of the filter under test. 
The tests for solarization did not detect any solarization under the limited set of conditions tested. One test was the spectrophotometry of exposed filters, in comparison with as-received filters. Solarization would be perceived as a change in the transmission spectrum after irradiation. We obtained spectra of filters after exposure to a single pulse of $\sim 1 \mathrm{~J} / \mathrm{cm}^{2}$. As seen in Figure 9, there is no sensible difference between this ("X") trace and several other traces obtained from unexposed samples. Unfortunately, schedule slippage at OSL caused our experiment to coincide with a power outage in building 298 (where the spectrophotometer was) and a trip to conduct an experiment at LLE (Rochester, NY). Thus, the spectrophotometer was not available immediately following the UV exposure of the filters. So, instead of obtaining the spectra "in real time," the exposed-filter scans were performed a week after the laser shots. Relaxation of the filter glasses could have occurred during the intervening period.
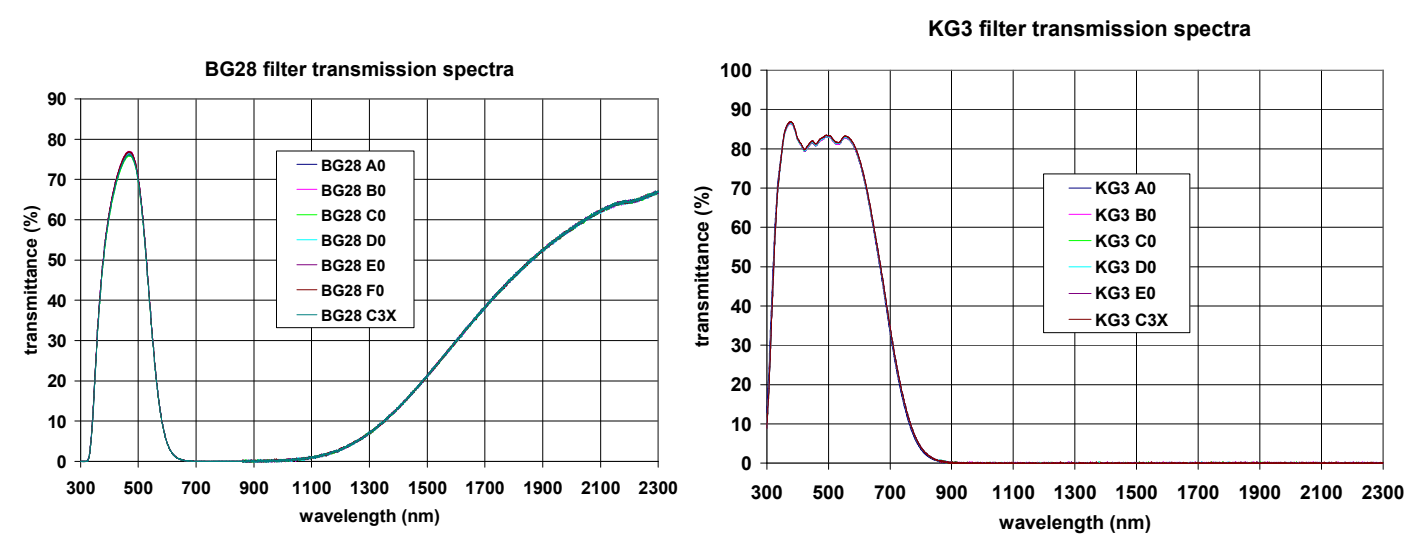

Fig. 9. Transmission spectra of BG28 and KG3 filters. The suffix " $X$ " on one curve for each filter type denotes exposure at $\sim 1 \mathrm{~J} / \mathrm{cm}^{2}$. Scans of exposed filters occurred roughly one week after the UV light dose. All other curves are for as-received filters. No solarization is evident.

Calorimeter readings can also serve as tests for changes in filter transmittance, but it is difficult to obtain a sufficiently high signal-to-noise ratio. Figure 10 presents results of calorimeter readings obtained during the calibration phase of the experiment. The energies actually striking the detectors were reduced by a factor of 134, using 2 uncoated fused silica wedges at 45 degrees, in order to fall within the dynamic range of the pyroelectric detectors used. (This dynamic range is stated by the manufacturer to be $1 \mu \mathrm{J}$ to $25 \mathrm{~mJ}$, but it is likely that ambient noise in OSL precludes measurements at the low end.) The input energy detector was calibrated against a NIST-traceable, calibrated 1" calorimeter placed at the sample plane. The output detector was then adjusted to match the input reading with no sample installed. Fig. 10(a) shows how the transmitted-energy reading tracks the incident energy over a wide range in the absence of a sample. But when this data is reduced to obtain the "transmittance" (Fig. 10(b)) it is evident that the noise level is high, with departures of $10 \%$ or more from the baseline. So, we did not observe useful trends to measure solarization effects with the sensitivity desired for the filter types (BG28, KG3, OG-550) tested. To make this apparatus work properly, higher pulse energies would have to strike the detectors, or the calorimeter electronics would need to reject noise better. 

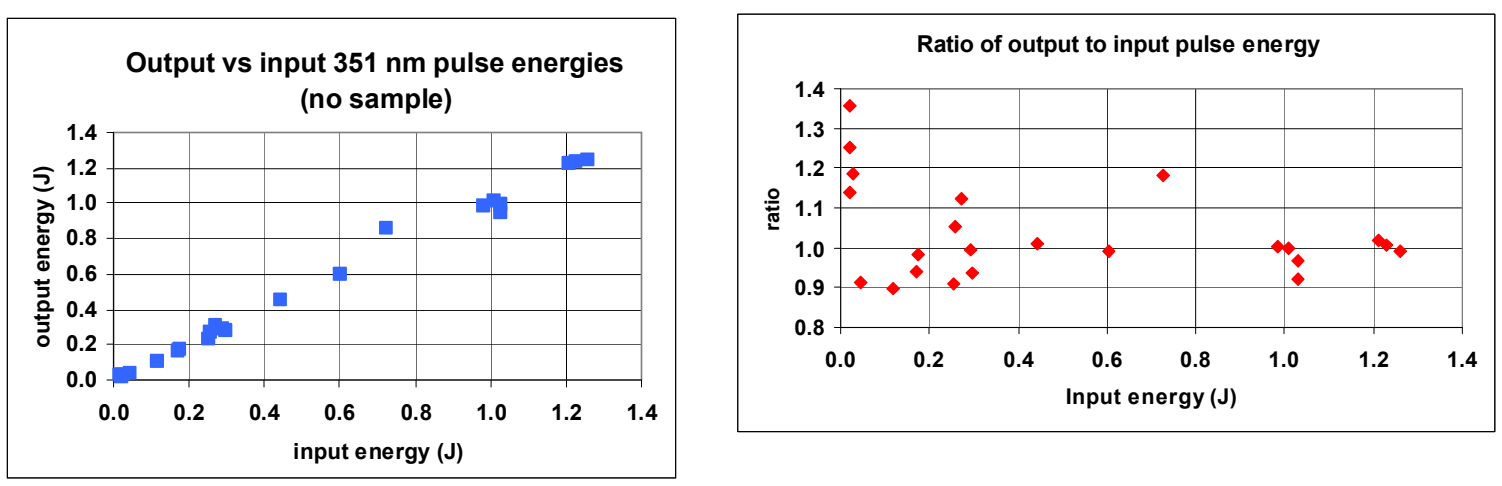

Fig. 10. Pyroelectric calorimeter data obtained with no sample in place. (a) Output tracks input fairly well, but there is scatter in the data. (b) Plot of "transmittance" (output divided by input) shows that the noise level is too high for successful detection of filter transmission changes.

The fluorescence test produced qualitative results that help understand the filter photophysics (see Discussion section). Spectrometer data showed strong signals for OG550 ("standard;" Fig. 11(a)) and for BG28 (Fig 11(b.)) These spectra have not had baselines subtracted, and there is a "tail" rising toward the IR in the BG28 spectrum. Because the signals were collected from the edge of the filters, the escaping light had to traverse $\sim 1 \mathrm{~cm}$ of filter material, causing some re-absorption of the luminescence. Hence we see a $550 \mathrm{~nm}$ "edge" at the filter cutoff wavelength in the OG-550 spectrum. The similar magnitudes of the OG-550 and BG28 signals imply a few-percent fluorescence quantum yield for the BG28 filter. (Refinement of the quantum-yield number requires a great deal of work calibrating the spectrometer sensitivity, collection efficiency, and absorbed energy, and is beyond the scope of this investigation.) This result constitutes evidence that the BG28 filter is not behaving purely as an attenuator. KG3 filters, on the other hand, never showed any fluorescence within the spectrometer's passband. In addition, the photodiode we set up to look at the edge of the filter under test did not show clear signals for any of the filters. It is likely that the filter edge was not properly imaged onto the photodiode's active area.
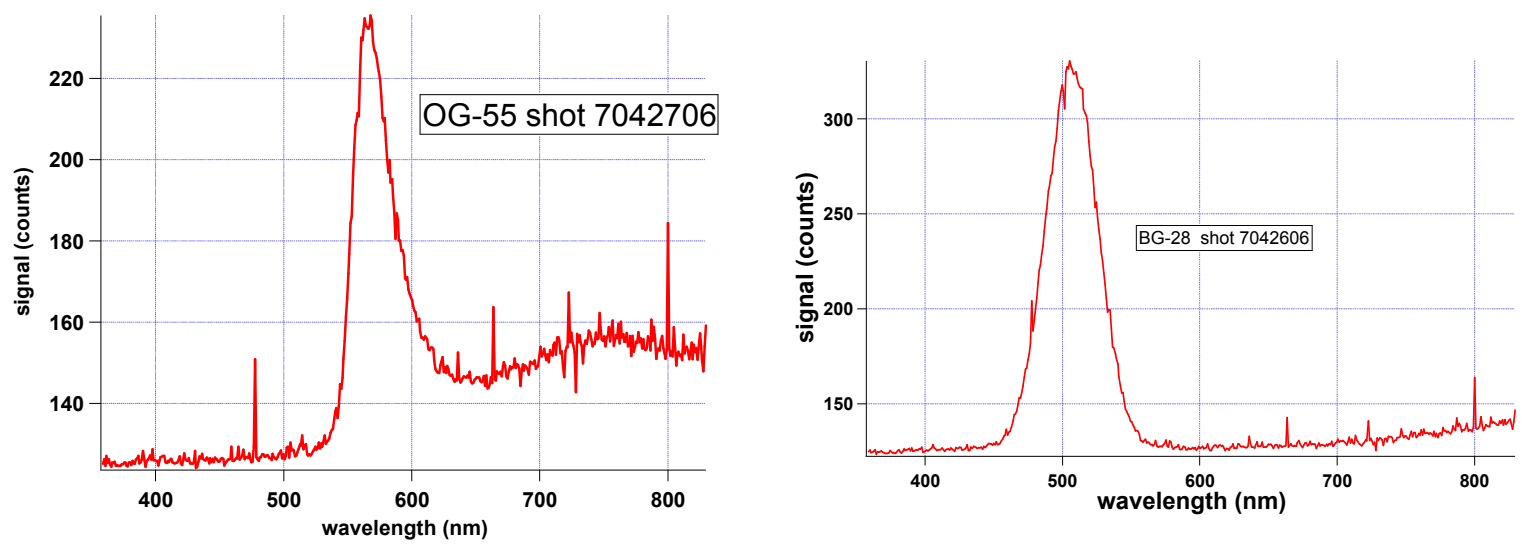

Fig. 11. Fluorescence spectra of (a) OG-550, the standard, and (b) BG28. The IR tail on the latter is an artifact, as are the sharp spikes in both spectra. Fluorescence is generally undesirable in an attenuator filter because it is a symptom of perturbation of the ground-state absorber population. 
The most important part of the experiment involved measuring and analyzing the pulse shapes of the incident and transmitted light signals, since they are a direct measure of pulse distortion. As a baseline test, signals were recorded with no sample (see Figure 12.) To create the plots in the figure, time and voltage offsets have been removed, and the amplitudes have been rescaled to overlay the traces. The effective bandwidths on the two signals are probably not exactly the same, so that the high frequency oscillations don't match perfectly. Yet, it is possible to match the traces to within a couple of percent. As mentioned previously, the relative height of the first "knee" depends on the laser energy setting.


Fig. 12. Two examples of overlaid incident and transmitted photodiode waveforms, in the absence of a sample, showing that the signals track accurately, enabling sensitive detection of pulse distortion.

When a KG3 filter was inserted into the path of the $351 \mathrm{~nm}$ light, no pulse distortion was detected, even at the high fluence of $\sim 1.41 \mathrm{~J} / \mathrm{cm}^{2}$ (Figure 13.) Based on the linearspectroscopic results, this makes sense - the filter does not attenuate much at $351 \mathrm{~nm}$ (see Fig. 9,) so not much energy is absorbed. The lack of fluorescence is a hint that the absorbing species are not getting depleted, nor are excited states (that could cause multiphoton absorption) being populated. So, there is no concern about using KG3 at $351 \mathrm{~nm}$.



Fig. 13. Incident and transmitted photodiode waveforms for KG3 glass at the high fluence of $\sim 1.41 \mathrm{~J} / \mathrm{cm}^{2}$. There is no detectable change in the pulse shape. 

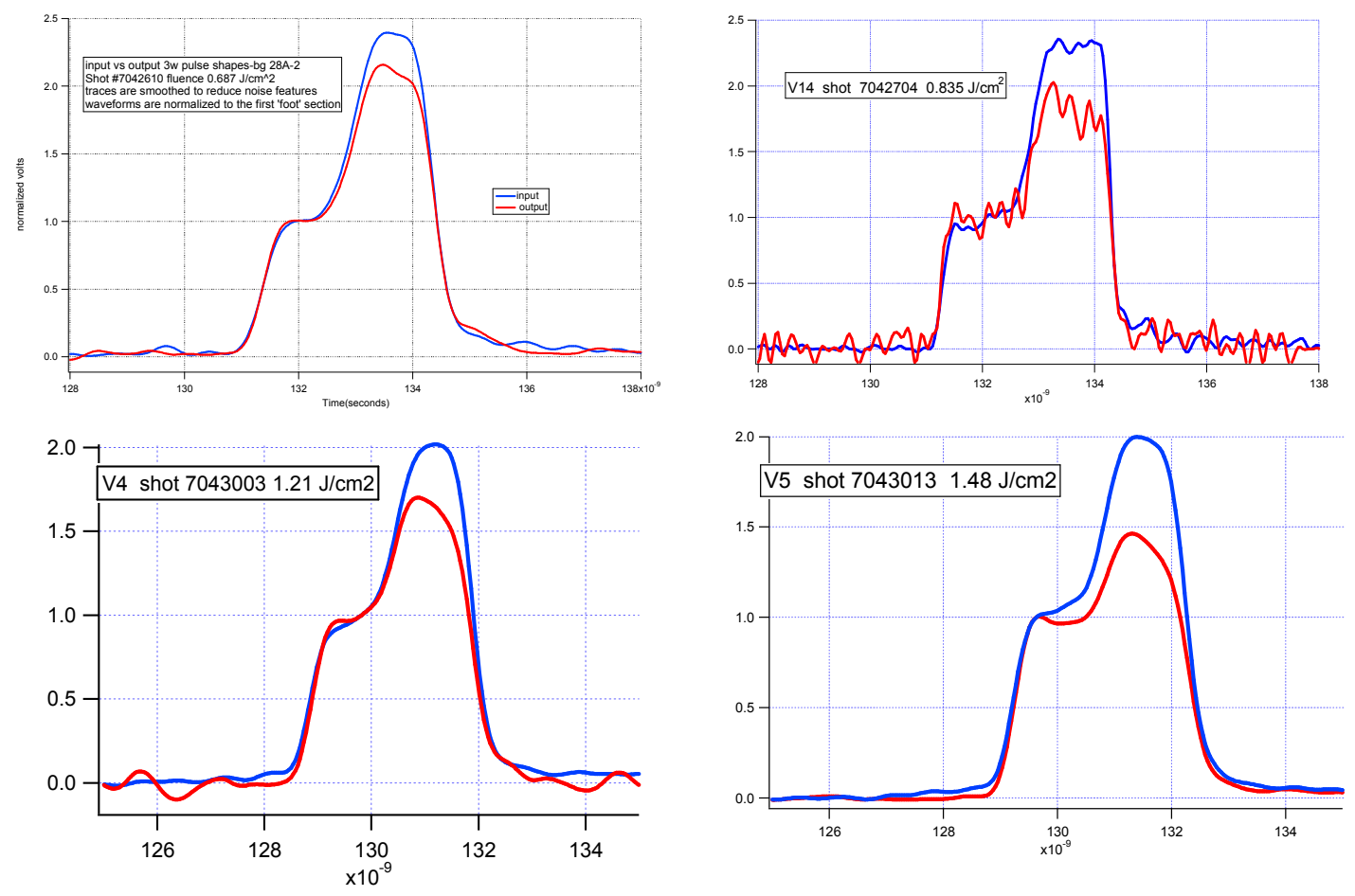

Fig. 14. Pulse distortion, or "droop", in BG28 filters is evident in the comparison between incident (blue) and transmitted (red) waveforms. The effect is stronger at higher fluences, and can be modeled to predict its severity under NIF conditions.

BG28 filters, on the other hand, do exhibit pronounced nonlinear effects. Figure 14 shows how the transmitted pulse (red) deviates increasingly from the incident pulse (blue) as time passes and more energy has been deposited in the filter. In each of the four panels, the traces have been rescaled and the initial "knee" has been aligned-it is assumed that before energy has been deposited, regardless of intensity, the filter retains its initial small-signal transmittance. Smoothing was performed on the traces in panels (a,) (c,), and (d.) The gradually-increasing shortfall in the transmitted-pulse signal is termed "droop." Droop is evidently more severe at higher fluence, as can be seen in the progression from $\sim 0.687 \mathrm{~J} / \mathrm{cm}^{2}$ (a) to $\sim 0.835 \mathrm{~J} / \mathrm{cm}^{2}$ (b) to $\sim 1.21 \mathrm{~J} / \mathrm{cm}^{2}$ (c) to $\sim 1.48$ $\mathrm{J} / \mathrm{cm}^{2}$ (d.) Modeling and quantifying the droop in order to predict the behavior under NIF operating conditions (nominally $0.04 \mathrm{~J} / \mathrm{cm}^{2}$ ) is the subject of section IV. The rationalization of the model is deferred to the Discussion section (VI.)

\section{BG28 Data Reduction, Modeling and the "Droop Parameter"}

We want to model the transmitted signal as a product of the input signal and the instantaneous filter transmittance:

$$
V_{\text {out }}(t)=T(t) \bullet V_{\text {in }}(t)
$$

Furthermore, the droop is seen to increase with absorbed fluence, so it makes sense to integrate the intensity and include a multiplicative proportionality constant (D.) Finally, 
for reasons explained in more detail below, we expect a Beer's-law (exponential) attenuation to describe the filter transmittance. The functional form we use to describe the time- and fluence-dependent droop is

$$
\frac{T(t)}{T_{0}}=\exp -D \int_{0}^{t} I\left(t^{\prime}\right) d t^{\prime}
$$

Here $T_{0}$ is the starting filter transmittance, and $\mathrm{I}(\mathrm{t})$ is the absorbed intensity; its time integral is thus a fluence.

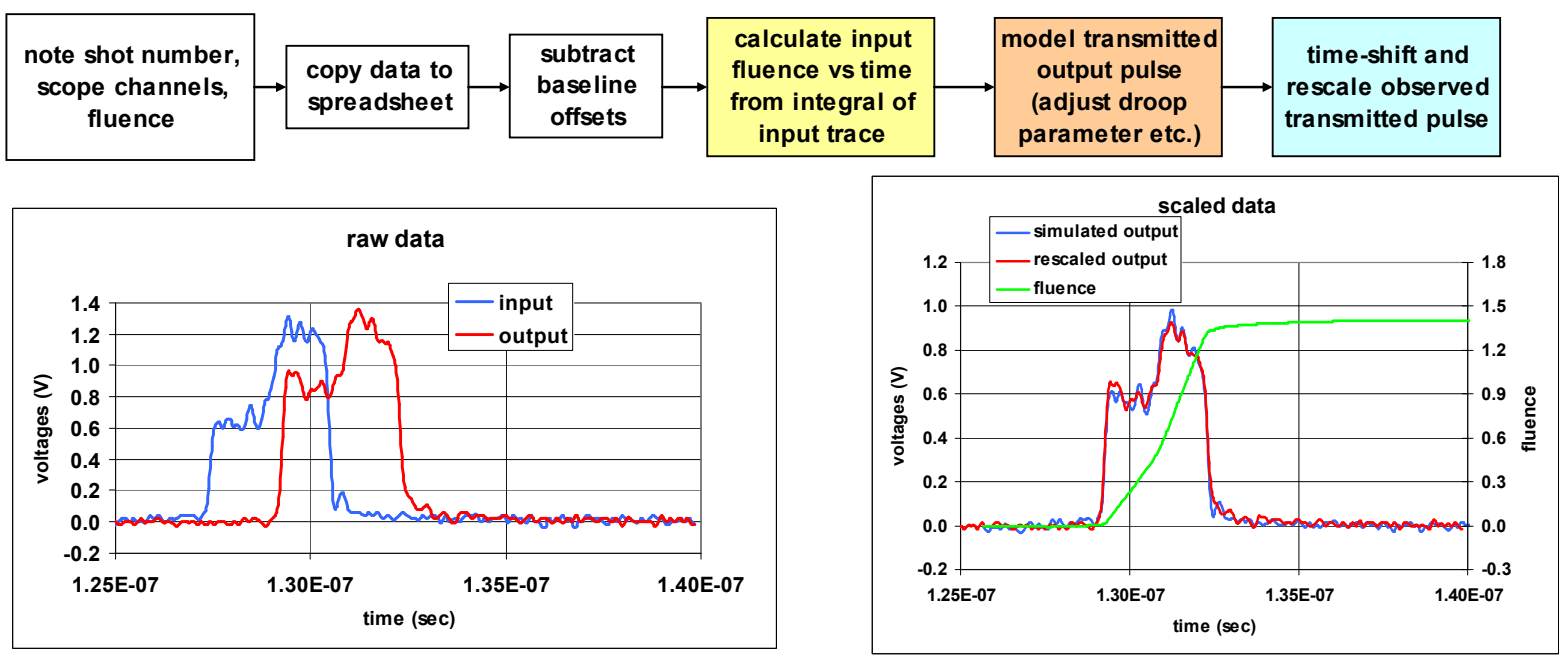

Fig 15. Fitting waveforms to obtain the "droop parameter" D. (a) Protocol followed to fit each pair of input/output photodiode traces. (b) Unsmoothed data from shot 7043013, whose nominal fluence was 1.48 $\mathrm{J} / \mathrm{cm}^{2}$, prior data processing. Blue - input pulse; red - transmitted pulse. (c) Photodiode signals with droop model applied. Green - elapsed fluence vs time. Red - transmitted pulse. Blue-simulated transmitted pulse. The value of $\mathrm{D}$ was $0.37 \mathrm{~cm}^{2} / \mathrm{J}$.

To obtain the value of D, we follow the protocol of Figure 15(a.) Because the photodiode waveforms (Fig. 15(b)) do not have absolute calibration, it is necessary to establish a fluence scale for the incident signal by equating its area to the value obtained from the calorimeter reading. Upon such normalization, the elapsed-fluence history can be calculated (see the green curve in Fig. 15(c.)) This value is then multiplied by D to calculate the transmittance $\mathrm{T}(\mathrm{t})$ as in equation (2.) Applying this factor to the input pulse allows comparison with the output pulse. The value of $\mathrm{D}$ is adjusted for the best fit - in Fig. 15(c,) D was set to $0.37 \mathrm{~cm}^{2} / \mathrm{J}$.

Table I lists the four shots analyzed to find values of $\mathrm{D}$. Upon averaging the results, the derived value for the filters tested is $\mathrm{D}=0.38 \pm 0.08 \mathrm{~cm}^{2} / \mathrm{J}$. 
Table I. Droop-parameter values derived from fits to BG28 filter data.

\begin{tabular}{|c|c|c|c|}
\hline OSL Shot Number & Igor file & $\begin{array}{c}\text { Average fluence } \\
\left(\mathrm{J} / \mathrm{cm}^{2}\right)\end{array}$ & $\begin{array}{c}\text { Droop Parameter (Fit) } \\
\left(\mathrm{cm}^{2} / \mathrm{J}\right)\end{array}$ \\
\hline 7042610 & V2 & 0.687 & 0.32 \\
\hline 7042704 & V14 & 0.835 & 0.52 \\
\hline 7043003 & V4 & 1.21 & 0.32 \\
\hline 7043013 & V5 & 1.48 & 0.37 \\
\hline average & & & $\mathbf{0 . 3 8} \pm \mathbf{0 . 0 8}$ \\
\hline
\end{tabular}

One effect we have not quantified is the error caused by the "top hat" approximation to the spatial intensity distribution. In reality, the observed signals are weighted by regions of above-average brightness. So, the perceived droop is stronger than it would be with a strictly-uniform distribution. Thus, the droop factor is over-estimated. See the Appendix for further discussion.

\section{Redesign of the Drive Diagnostic optical system to Mitigate "Droop"}

To estimate the pulse distortion of the Drive Diagnostic as designed, it was necessary to scale the droop parameter appropriately for the BG28 filter to be used. This is simply done by considering that the strength of the droop is proportional to the absorbed fluence. For a filter with internal transmittance $T_{\text {int }}$ and surface reflectivity $R$, the "fraction absorbed" FA is roughly

$\mathrm{FA} \approx(1-\mathrm{R})\left(1-\mathrm{T}_{\text {int }}\right)\left(1+\mathrm{R} \bullet \mathrm{T}_{\text {int }}\right)$.

For the stock filter we used, this factor is approximately 0.733 , and for the WF2 filter in the Drive Diagnostic, it is 0.97 . The ratio of these numbers, 1.32 , is used to obtain the scaled droop parameter of

$\mathrm{D}($ drive diagnostic $)=0.50 \pm 0.11 \mathrm{~cm}^{2} / \mathrm{J}$.

For the design-maximum sampled fluence of $0.04 \mathrm{~J} / \mathrm{cm} 2$, this would lead to a pulse-end droop of $[1-\exp -\{(0.50)(0.04)\}]=2 \%$.

Figure 16 shows a sample calculation of droop error for a 1.8 MJ inner-cone Haan pulse. The parameter values used to represent the Drive Diagnostic in this simulation were:

- Pickoff fraction $2.54 \times 10^{-6}$

- Spot area $0.7225 \mathrm{~cm}^{2}$

- Resulting fluence on BG28 WF2 filter $0.033 \mathrm{~J} / \mathrm{cm}^{2}$.

The fractional error (green trace, right-hand scale) is negligible during the first $15 \mathrm{nsec}$ of the pulse but reaches $\sim 1 \%$ near the peak of the pulse and exceeds $1.6 \%$ at the end of the pulse. 


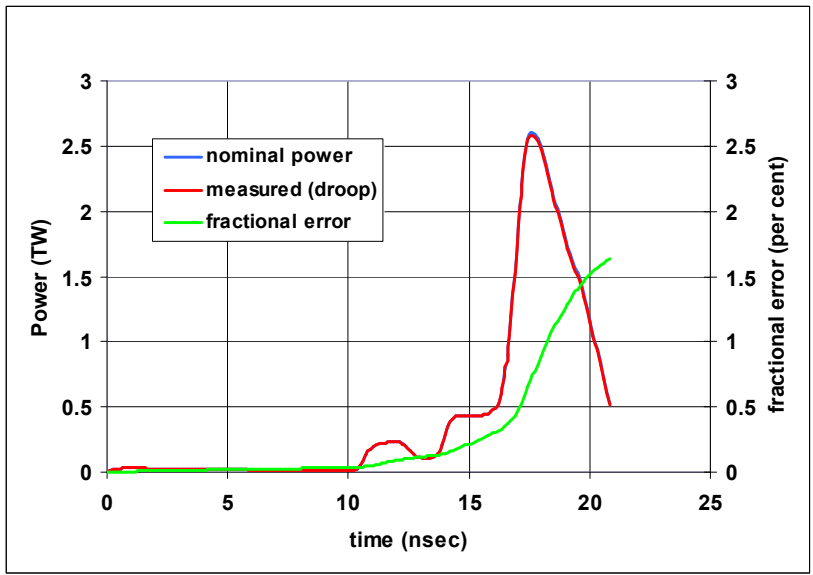

Fig. 16. Simulation of droop observable in a diagnostic signal when a $1.8 \mathrm{MJ}$ inner-cone Haan pulse is applied. The green trace (right-hand scale) indicates that an error of $1.6 \%$ builds up during the pulse. This large error mandated some design changes to reduce the operating fluence of the BG28 filters in the diagnostic.

Diagnostic-signal errors in the $1-2 \%$ range were considered excessive. Various mitigation strategies exist, but it was decided to reduce the fluence on the WF2 (and downstream) filters. The design-maximum fluence was to be reduced from $0.04 \mathrm{~J} / \mathrm{cm}^{2}$ to $0.01 \mathrm{~J} / \mathrm{cm}^{2}$, which would reduce the peak signal error to $0.5 \%$. This would be accomplished by reducing the pickoff fraction from the Grating Debris Shield from 0.003 to 0.002 and reducing the reflectances of the two turning mirrors WM8 and WM14 from $15 \%$ to $8.5 \%$ (maximum.).

\section{Discussion Regarding Photophysics of "Droop"}

A diagram consistent with the droop model of equation (2) is shown in Figure 17 (a.) The filter's small-signal transmittance is governed by ground-state absorption by a species whose $351 \mathrm{~nm}$ absorption cross section is $\sigma_{\mathrm{GSA}}$ and whose number density is $\mathrm{N}_{1}$. Absorption of ultraviolet light pumps the population into a metastable level via fast radiationless decay. The population $\mathrm{N}_{2}$ in this level builds up as fluence is absorbed, leading to an induced absorption with excited-state cross section $\sigma_{\mathrm{ESA}}$. It is assumed that excited-state absorption does not populate additional metastable levels. In this scenario, the total population $\left(\mathrm{N}_{1}+\mathrm{N}_{2}\right)$ is conserved. The transmittance as a function of level populations then becomes

$$
T=\exp -\left(N_{1} \sigma_{G S A} L+N_{2} \sigma_{E S A} L\right)
$$


For the BG28 filter at $351 \mathrm{~nm}, \sigma_{\mathrm{ESA}}>\sigma_{\mathrm{GSA}}$ and the filter transmission decreases as light is absorbed. Figure $17(b-d)$ illustrates this schematically by showing how the separate contributions to the extinction coefficient evolve with elapsed fluence. The slope of the total extinction coefficient with respect to elapsed fluence is simply D, the droop parameter.
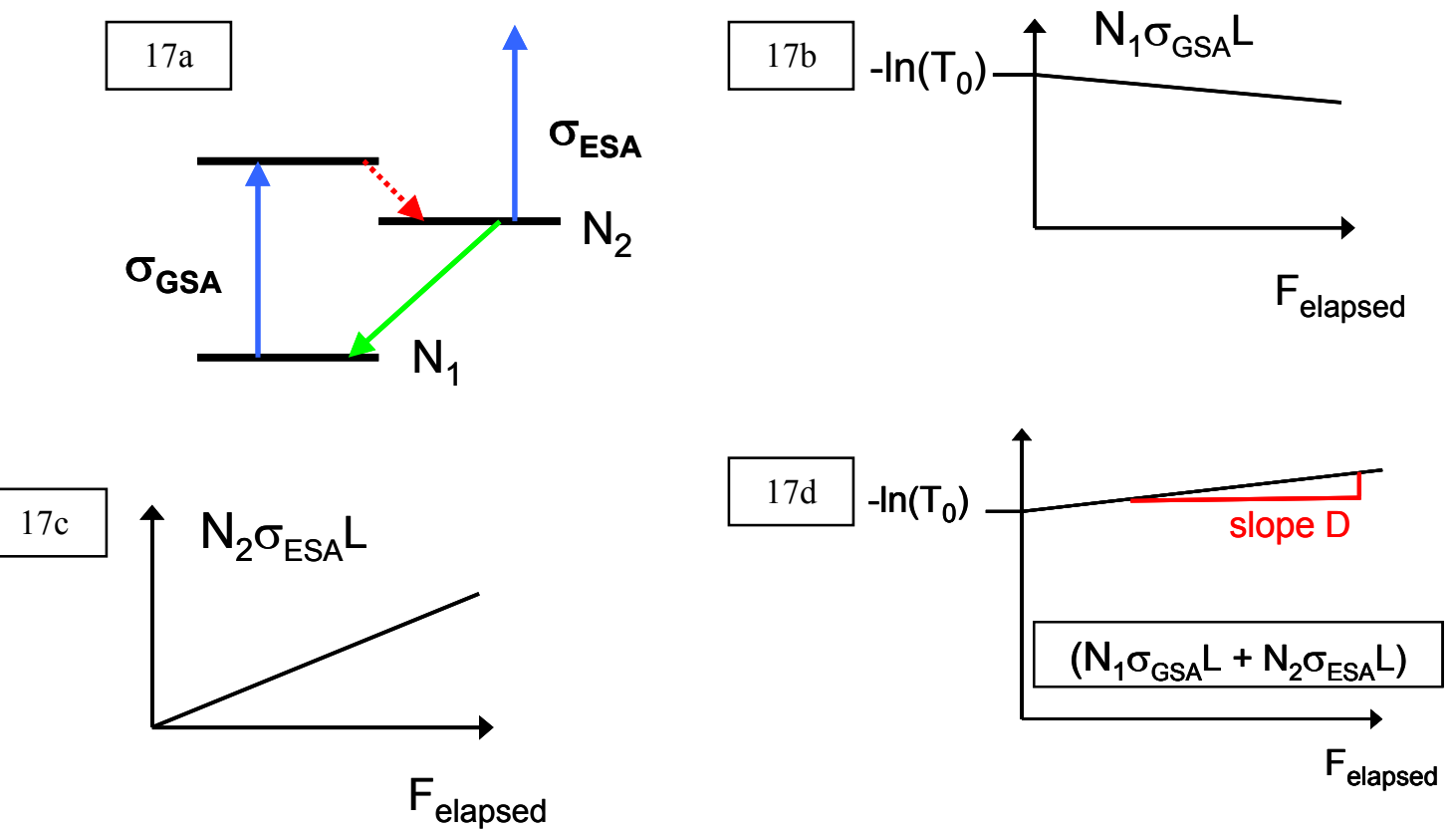

Fig. 17. (a) Ground-state depletion / excited-state absorption model of droop. The ESA cross section exceeds the GSA cross section. $(b-d)$ evolution of the ground-state, excited-state, and overall contributions to the extinction coefficient as fluence is absorbed.

Copper is one of the main dopants used in blue-green glasses, including BG28. It almost certainly exists in two valence states-divalent $\left(\mathrm{Cu}(\mathrm{II}) ; \mathrm{Cu}^{2+}\right)$ and monovalent $(\mathrm{Cu}(\mathrm{I})$; $\mathrm{Cu}^{+}$) with the partition depending on details of glass chemistry and melt conditions. [12, 13] As Figure 18(a) shows, divalent copper gives rise to a strong absorption in the red and near-IR, $[12,14,15]$ which we perceive as "blue" owing to the color of the unabsorbed light. Monovalent copper, on the other hand, is a UV absorber [14 - 16] and may appear colorless in some hosts. It is probably the species responsible for the $351 \mathrm{~nm}$ absorption. The $\mathrm{Cu}^{+}$level scheme $[17,18]$ of Figure 18(b) shows how absorption on the "allowed" ${ }^{1} \mathrm{E}_{\mathrm{g}} \leftarrow{ }^{1} \mathrm{~A}_{\mathrm{g}}$ transition can be followed by radiationless decay populating the ${ }^{3} \mathrm{E}_{\mathrm{g}}$ triplet level, which exhibits a "forbidden" transition back to the ground level. This latter transition is observed as phosphorescence, whose lifetime has been reported as tens of $\mu$ sec. $[17,19,20]$ This diagram is in accordance with that of Fig. 17(a.) Interestingly, copper-doped glass luminescence was once imagined as the basis for a blue-green laser [21] and has also been considered as a means of imaging ultraviolet laser beams with high spatial fidelity. [22] 

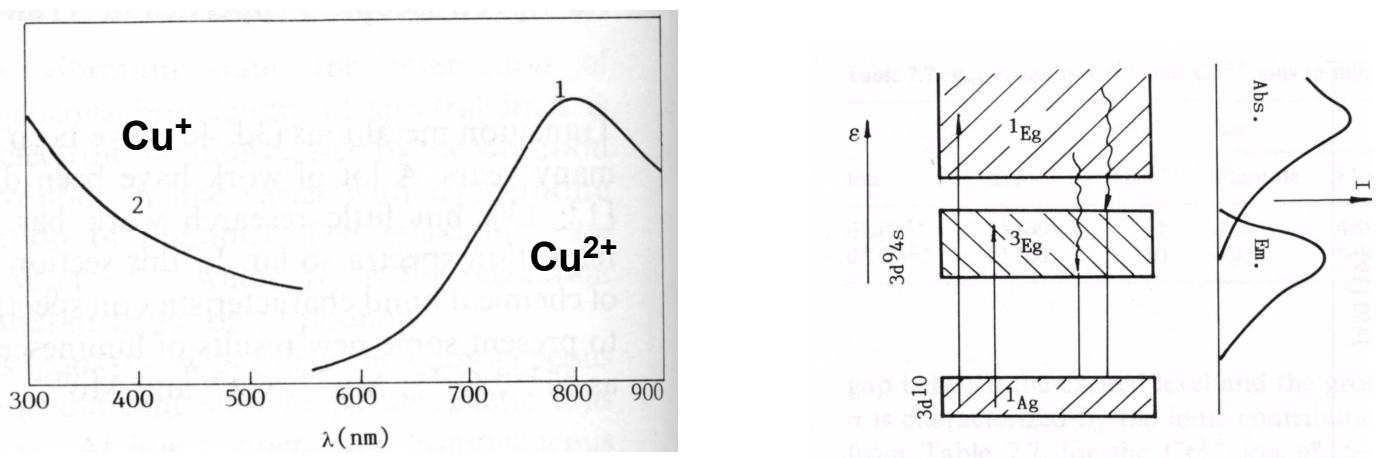

Fig. 18 (after Gan. [17]) (a) Absorption spectra of divalent (red) and monovalent (UV) copper in some host. (b) $\mathrm{Cu}+$ level scheme and luminescence pathway (in accordance with the droop model described herein.)

More work on BG28 solarization may be warranted. It is not clear whether the lifetimeof-NIF integrated dose of UV light on the BG28 filters will unacceptably perturb their spectra. The data of Figure 3 can serve as a guide for an extrapolation, but a nonlinear intensity dependence of the solarization rate and/or a saturation of the effect could reduce confidence in such predictions. In figure 3, the maximum applied fluence (after over 100 shots) is $\sim 100 \mathrm{~J} / \mathrm{cm}^{2}$, which corresponds to $\sim 10^{4} \mathrm{NIF}$ shots delivering $0.01 \mathrm{~J} / \mathrm{cm}^{2}$ apiece to the Drive Diagnostic filters. Based on this simple arithmetic, drift in the filter properties would be expected during NIF's lifetime. It would be prudent to characterize several of the WF2 filters after several years of use in the NIF.

Another topic of potential interest is the behavior of BG28 filters at $527 \mathrm{~nm}$. Given the energy level distribution of $\mathrm{Cu}^{2+}$ ions and the known spectroscopy, it is unlikely that nonlinear effects will be important at this wavelength and contemplated fluence level. Otherwise, intensity-dependent transmission phenomena would most likely already have been reported.

\section{Acknowledgements}

Paul Wegner provided the primary motivation and guidance for this study and made the resources available. We are grateful to Ken Manes for calling our attention to the problem of filter characterization, and to him, Bruno van Wonterghem, and Rich Zacharias for discussions of "measles." John Adams generously furnished manuscripts, viewgraphs, and data regarding a previous solarization study and nonlinear-absorption data. Mike Borden forwarded a BG28 transmission spectrum for our use. Larry Siegel and Simon Cohen were involved in discussions concerning various DrD design and filter choices.

Mike Nichols and Kathy Weems speedily dealt with filter procurements. Wren Carr consulted on the configuration the experiments would take in OSL. The OSL staff (Ron Luthi, Drew Willard, Carmelo Martinez, Jim Vickers, and Frank Ravizza) constructed and calibrated our experiment in short order. Steve Letts graciously allowed the use of a 
Lambda 9 spectrophotometer. Keith Kanz loaned us "Pen-Ray" spectral-calibration lamps, and Scott Mitchell provided filter fluorescence spectra.

Steven Yang shared viewgraphs and experience gained from working on PDS. Chris Haynam and Mike Shaw furnished NIF laser pulse shapes and power-balance specifications that enabled estimates of the severity of "droop."

Dr. Joseph S. Hayden (Schott Glass Technologies) shared his expertise about the properties and production of blue-green and copper-doped filters, and called our attention to pertinent literature.

\section{Appendix on Fluence Distributions}

It was mentioned above that "hot spots" contribute dis-proportionately to the transmittedbeam photodiode signal, and that they also experience stronger "droop" than would be expected based on the top-hat-model average fluence. Because fluence maps (Fig. 8) have been recorded for the laser shots we took in OSL, one could contemplate further data reduction and modeling to account for the actual fluence distribution. This Appendix presents equations showing how the fluence-map data could be used in the fitting procedure (Fig. 15) to find improved values for D, the droop parameter.

First, we assume that the spatial and temporal variables can be separated, so that

$d^{2} E / d A d t=I(\vec{r}, t) \equiv P(t) G(\vec{r})$

Where $\mathrm{P}(\mathrm{t})$ being the instantaneous power and $\mathrm{G}$ being the normalized fluence distribution (which has units of inverse area:)

$$
\int_{\text {spot }} G(\vec{r}) d A \equiv 1
$$

It automatically follows that

$$
\begin{aligned}
& \iint_{\text {pulse }} I(\vec{r}, t) d A d t=E_{\text {pulse }} \quad \int_{\text {spot }} I(\vec{r}, t) d A=P(t) \quad(\mathrm{A}-3 \mathrm{a}, \mathrm{b}, \mathrm{c}) \\
& \quad \int_{0}^{t_{0}} I(\vec{r}, t) d t=G(\vec{r}) \int_{0}^{t_{0}} P(t) d t
\end{aligned}
$$

The spot-averaged values of the distribution function and elapsed fluence are then

$$
\begin{aligned}
\bar{G} & =A_{\text {eff }}^{-1} \\
\bar{F}\left(t_{0}\right) & =\bar{G} \int_{0}^{t_{0}} P(t) d t=A_{\text {eff }}^{-1} \int_{0}^{t_{0}} P(t) d t
\end{aligned}
$$


When we seek to compare "incident" and "transmitted" photodiode signals, we integrate over the laser spot. When no sample is present, we have (defining a photodiode responsivity R:)

$$
V\left(t_{0}\right)=R \int_{\text {spot }} I\left(\vec{r}, t_{0}\right) d A=R P\left(t_{0}\right)
$$

With a sample in place, we introduce the spatially-dependent transmission factor to obtain

$$
V\left(t_{0}\right)=R \int_{\text {spot }} I(\vec{r}, t) \bullet\left(\exp -D \int_{0}^{t_{0}} I(\vec{r}, t) d t\right) d A
$$

If the fluence is assumed to be spatially uniform, we recover the previously-used formula for the calculated signal (eqs 1, 2:)

$$
V\left(t_{0}\right)=R P\left(t_{0}\right) \bullet \exp -D \bar{F}\left(t_{0}\right)
$$

Otherwise, (A-7) can be expanded as a series and evaluated with knowledge of G(r) and a choice of D:

$V\left(t_{0}\right)=R P\left(t_{0}\right)[\overbrace{\int_{\text {spot }} G(\vec{r}) d A}-\overbrace{D \int_{\text {spot }} G^{2}(\vec{r}) d A \bullet \int_{0}^{t_{0}} P(t) d t+\frac{D^{2}}{2} \int_{\text {spot }} G^{3}(\vec{r}) d A}^{1} \overbrace{\left(\int_{0}^{t_{0}} P(t) d t\right)^{2}}^{\text {Higher-order terms give extra weight to "hot spots" }}]$ (A-9)

D would then be varied, as before, to optimize agreement between the observed and calculated transmitted waveforms.

\section{References and bibliography}

[1] Optical Glass Filters (Schott Glaswerke, Mainz, Germany,) section 5.5

("Transmission changes caused by intense ultraviolet radiation.")

[2] W. L. Smith, "Two-photon absorption and solarization in UV glasses," 1982 Laser Program Annual Report, pg. 7-34.

[3] F. Rainer, "How safe are your laser goggles? - revisited," memo LRD 83/219/3441D, November 30, 1983.

[4] D. W. Myers, "Filter attenuation and solarization," memo NOVA ACD 86-078, June 9, 1986. 
[5] C. J. Stolz, J. A. Menapace, F. Y. Genin, P. R. Ehrmann, P. E. Miller, and G. T. Rogowski, "Influence of BK7 substrate solarization on the performance of hafnia and silica multilayer mirrors," UCRL-JC-148404 (2002.)

[6] Ken Manes and Rich Zacharias, private communications.

[7] J. J. Adams, T. McCarville, J. Bruere, J. McElroy, and J. Peterson, "Effects of nonlinear absorption in BK-7 and color glasses at 355 nm," LMOT presentation, 19 Nov 03.

[8] R. Debnath and J. Chaudhuri, "Coordination structure and the bonding properties of UV-laser-induced photo-generated $\mathrm{Cu}(\mathrm{II})$ in a monovalent copper containing silica glass," Chem. Phys. Lett. 217, 357 (1994.)

[9] H. Ebendorff-Heidepriem and D. Ehrt, "UV radiation effects in fluoride phosphate glasses,” J. Non-Crystalline Solids 196, 113 (1996.)

[10] ref [1]; see entry for BG 28 (pg. 52)

[11] M. Wittman and A. Penzkofer, "Spectroscopic characterization of semiconductor doped colour filter glasses," Opt. Quantum Elec. 27, 705 (1995.)

[12] S. P. Singh and A. Kumar, "Molar extinction coefficients of the cupric ion in silicate glasses,” J. Mat. Sci. 30, 2999 (1995.)

[13] R. S. Singh and S. P. Singh, "Thermodynamic activity of cuprous-cupric redox oxides in alkali copper silicate glasses," J. Mat. Sci. 38, 1551 (2003.)

[14] P. C. Schulz, "Optical absorption of the transition elements in vitreous silica," J. Amer. Ceram. Soc. 67, 309 (1974.)

[15] Theodore L. Brown, H. Eugene LeMay, Jr., and Bruce E. Bursten, Chemistry: The Central Science ( $7^{\text {th }}$ ed.) (Prentice Hall, Upper Saddle River, New jersey, 1997,) pg. 890.

[16] R. Debnath and S. Kumar, "Dual luminescence of $\mathrm{Cu}^{+}$in glass," J. Non-Crystalline Solids 123, 271 (1990.)

[17] Gan Fuxi, Optical and Spectroscopic Properties of Glass, (Springer, Berlin, 1992,) pg. 160.

[18] R. Debnath and S. K. Das, "Site-dependent luminescence of $\mathrm{Cu}^{+}$ions in silica glass," Chem. Phys. Lett. 155, 52 (1989.)

[19] Liu Huimin and Gan Fuxi, "Luminescence of $\mathrm{Cu}^{+}$ions in phosphate glass," J. NonCrystalline Solids 80, 447 (1986.) 
[20] R. Debnath, "On the excitation of the ${ }^{3} \mathrm{E}$ luminescent state of $\mathrm{Cu}^{+}$ions in glass," J. Luminesc. 43, 375 (1989.)

[21] "Copper-doped laser glass is proposed for tunable output at 400 to $800 \mathrm{~nm}$," Laser Focus, May 1981,pg. 22; L. G. DeShazer, "Cuprous ion doped crystals for tunable lasers," in Tunable Solid State Lasers, P. Hammerling, A. B. Budgor, and A. Pinto, eds. (Springer, Berlin, 1985,) pg 91.

[22] A. V. Deniz and J. A. Stamper, "A comparison of glass fluorescers used to measure a pulsed ultraviolet image at F/2,” Rev. Sci. Inst. 72, 1993 (2001.) 